\title{
NuSTAR observations of the black holes GS 1354-645: Evidence of rapid black hole spin
}

El-Batal, A. M.; Miller, J. M.; Reynolds, M. T.; Boggs, S. E.; Christensen, Finn Erland; Craig, W. W.; Fuerst, F.; Hailey, C. J.; Harrison, F. A.; Stern, D. K.

Total number of authors:

13

Published in:

The Astrophysical Journal Letters

Link to article, DOI:

10.3847/2041-8205/826/1/L12

Publication date:

2016

Document Version

Publisher's PDF, also known as Version of record

Link back to DTU Orbit

Citation (APA):

El-Batal, A. M., Miller, J. M., Reynolds, M. T., Boggs, S. E., Christensen, F. E., Craig, W. W., Fuerst, F., Hailey, C. J., Harrison, F. A., Stern, D. K., Tomsick, J., Walton, D. J., \& Zhang, W. W.'(2016). NuSTAR observations of the black holes GS 1354-645: Evidence of rapid black hole spin. The Astrophysical Journal Letters, 826(1), [L12]. https://doi.org/10.3847/2041-8205/826/1/L12

\section{General rights}

Copyright and moral rights for the publications made accessible in the public portal are retained by the authors and/or other copyright owners and it is a condition of accessing publications that users recognise and abide by the legal requirements associated with these rights.

- Users may download and print one copy of any publication from the public portal for the purpose of private study or research.

- You may not further distribute the material or use it for any profit-making activity or commercial gain

- You may freely distribute the URL identifying the publication in the public portal 


\author{
A. M. El-Batal ${ }^{1}$, J. M. Miller ${ }^{1}$, M. T. Reynolds ${ }^{1}$, S. E. Boggs ${ }^{2}$, F. E. Chistensen ${ }^{3}$, W. W. Craig ${ }^{2,4}$, F. Fuerst $^{5}$, C. J. Hailey ${ }^{6}$,

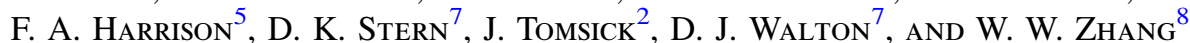 \\ ${ }^{1}$ Department of Astronomy, University of Michigan, 1085 S University Avenue, Ann Arbor, MI 48109, USA; jonmm@umich.edu \\ ${ }^{2}$ Space Sciences Laboratory, University of California, 7 Gauss Way, Berkeley, CA 94720-7450, USA \\ ${ }^{3}$ Danish Technical University, Lungby, Denmark \\ ${ }^{4}$ Lawrence Livermore National Laboratory, Livermore, CA 94550, USA \\ ${ }^{5}$ Cahill Center for Astronomy and Astrophysics, California Institute of Technology, 1200 East California Boulevard, Pasadena, CA 91125, USA \\ ${ }^{6}$ Columbia Astrophysics Laboratory and Department of Astronomy, Columbia University, 550 West 120th Street, New York, NY 10027, USA \\ ${ }^{7}$ Jet Propulsion Laboratory, California Institute of Technology, 4800 Oak Grove Drive, Pasadena, CA 91109, USA \\ ${ }^{8}$ NASA Goddard Space Flight Center, Greenbelt, MD 20771, USA \\ Received 2016 May 18; revised 2016 June 30; accepted 2016 July 1; published 2016 July 19
}

\begin{abstract}
We present the results of a NUSTAR study of the dynamically confirmed stellar-mass black hole GS 1354-645. The source was observed during its 2015 "hard" state outburst; we concentrate on spectra from two relatively bright phases. In the higher-flux observation, the broadband $N u S T A R$ spectra reveal a clear, strong disk reflection spectrum, blurred by a degree that requires a black hole spin of $a=c J / G M^{2} \geqslant 0.98$ ( $1 \sigma$ statistical limits only). The fits also require a high inclination: $\theta \simeq 75(2)^{\circ}$. Strong "dips" are sometimes observed in the X-ray light curves of sources viewed at such an angle; these are absent, perhaps indicating that dips correspond to flared disk structures that only manifest at higher accretion rates. In the lower flux observation, there is evidence of radial truncation of the thin accretion disk. We discuss these results in the context of spin in stellar-mass black holes, and inner accretion flow geometries at moderate accretion rates.
\end{abstract}

Key words: accretion, accretion disks - black hole physics - X-rays: binaries

\section{INTRODUCTION}

Low-mass X-ray binaries are binary systems comprised of a compact object accreting matter from a low-mass companion star. The accretion disk is luminous across the electromagnetic bandpass, but it peaks in the X-ray band. The hot corona contributes in the hard X-ray band. Without a need for strong bolometric corrections, X-ray studies of these sources can accurately constrain the geometry of the inner accretion flow and its energetic properties (for a review of stellar-mass black holes in such settings, see Remillard \& McClintock 2006). Owing to their proximity, Galactic binaries are excellent laboratories for probing the extreme gravitational effects of black holes.

Astrophysical black holes can be fully described by their mass and "spin" (dimensionless angular momentum; $a=c J / G M^{2}$, where $-1 \leqslant a \leqslant 1$ ). The location of the innermost stable circular orbit (ISCO) around the black hole depends on the spin parameter. For a Schwarzschild black hole (zero spin), the radius of the the ISCO is at $R_{\mathrm{ISCO}}=6 \mathrm{GM} / \mathrm{c}^{2}$. In an extreme Kerr black hole (maximally spinning), $R_{\mathrm{ISCO}} \simeq 1 G M / c^{2}$ (e.g., Bardeen et al. 1972).

Hard X-rays produced via Comptonization and/or sychrotron in the corona are "reflected" from the accretion disk, and this effect can be used to measure the spin of both stellar-mass and supermassive black holes (for recent reviews, see, e.g., Miller 2007; Reynolds 2014; Miller \& Miller 2015). The reflection spectrum is "blurred" by the strong Doppler shifts and gravitational redshifts close to the black hole, effectively tracing the radius of the ISCO and the spin of the black hole. Resolution is important in such studies, but sensitivity and a broad spectral bandpass are also very important. NuSTAR (Harrison et al. 2013) has unprecedented sensitivity in the 3-79 keV band and does not suffer from distorting effects such as photon pile-up; it is an ideal mission for studies of reflection and black hole spin (see, e.g., Miller et al. 2013a, 2013b; Risaliti et al. 2013; King et al. 2014; Parker et al. 2014; Tomsick et al. 2014; Furst et al. 2015).

Other important measurements can be obtained through reflection modeling. Among these are the inclination of the innermost accretion disk, though models can also measure the ionization of the inner disk, and can also constrain elemental abundances. In the best cases, the size of the hard X-ray corona can even be constrained (e.g., Miller et al. 2015). Progress has not only relied upon improved X-ray instrumentation, but also improved reflection models. In particular, relxill (e.g., Garcia et al. 2013; Dauser et al. 2014) offers many advantages over prior models, including internal relativistic blurring and angle-dependent scattering calculations.

The object of this work, GS 1354-645, is a binary system comprising a dynamically confirmed black hole of mass $M_{\mathrm{BH}} \geqslant 7.6(7) M_{\odot}$ (Casares et al. 2009) and a low-mass stellar companion. It was first detected using the All Sky Monitor aboard the Ginga satellite during an outburst in 1987 (Kitamoto et al. 1990). The last outburst of this source was detected using RXTE in 1997 (e.g., Revnivtsev et al. 2000; Brocksopp et al. 2001; also see Reynolds \& Miller 2013). The distance to the source is not well-constrained, with estimates lying between 25 and $61 \mathrm{kpc}$ (Casares et al. 2009).

Monitoring with the Swift/BAT detected a new outburst of GS 1354-645 in 2015 June (Miller et al. 2015). Better-known, recurrent sources like GX 339-4 often show multiple spectral states, but GS 1354-645 is interesting in that only the "hard" state was observed in its last outburst. GS 1354-645 may therefore belong to a small sub-class of black hole transients including the better-known GRO J0422+32 and XTE J1118 +480 (Brocksopp et al. 2001). Disk reflection was clearly required in RXTE spectra of the 1997 outburst of GS 1354 -645 (Revnivtsev et al. 2000), potentially indicating a means 


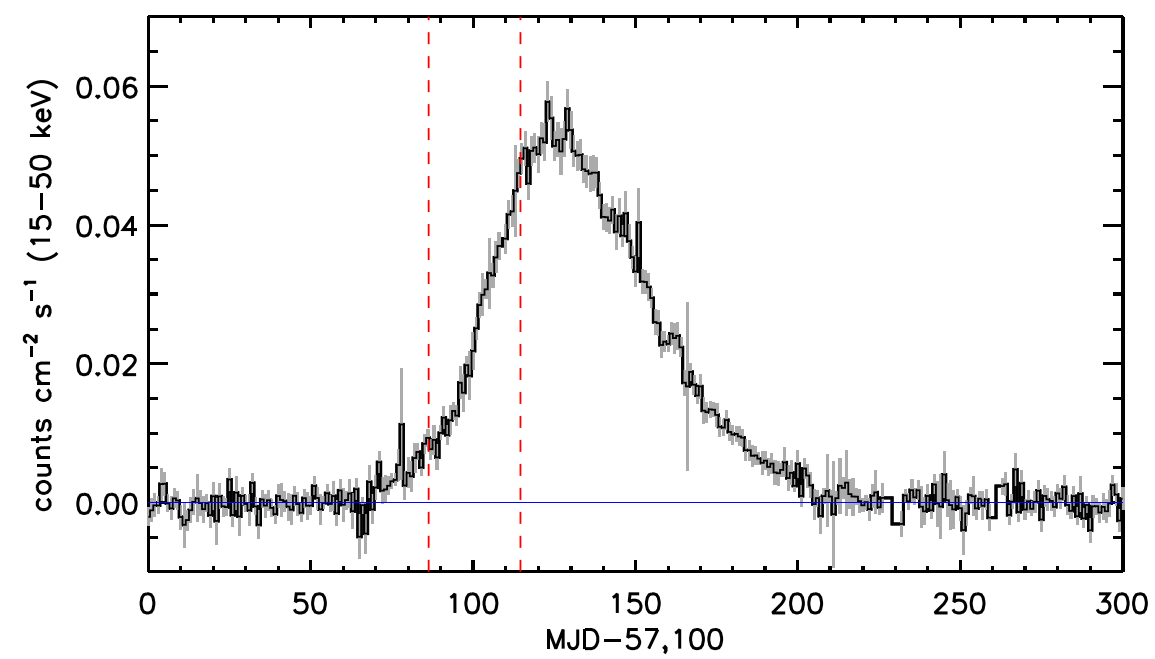

Figure 1. The Swift/BAT light curve of the 2015 outburst of the black hole GS $1354-645$. The data points represent the average flux over a span of one day. The red, dashed, vertical lines indicate the start time of the two NuSTAR observations considered in this work, referred to as Obs. 1 and Obs. 2. The outburst is qualitatively consistent with a fast rise, exponential decay (FRED) profile.

to study the black hole and innermost accretion flow in a "hard state" transient. We therefore requested observations with NUSTAR.

\section{DATA REDUCTION}

We obtained two NuSTAR observations of GS 1354-645 during its 2015 outburst. The first observation was made on 2015 June 13 (hereafter Obs. 1), starting at 06:56:07 (UT). It achieved a net exposure of $24 \mathrm{ks}$. The second observation (hereafter Obs. 2) was made roughly one month later, on 2015 July 11, starting at 13:41:08 (UT). Obs. 2 achieved a net exposure of $29 \mathrm{ks}$.

Data reduction was performed using the routines in HEASOFT version 6.16 and the associated NuSTAR calibration files (version 20150316). The nuproducts routine was run to extract source light curves, spectra, and responses, as well as background spectra. FPMA and FPMB source events were extracted using a 148 arcsec (radius) circle, centered on the source. Background events were extracted from a region of equivalent size in a source-free region.

\section{ANALYSIS AND RESULTS}

Figure 1 shows the light curve of the entire outburst, based on public monitoring observations made with the Swift/BAT. In qualitative terms, the outburst has a "fast rise, exponential decay" or FRED profile. The points at which Obs. 1 and Obs. 2 were made are indicated. Observation 2 was obtained at a flux $\sim 5$ times higher than Obs. 1; this is reflected in the signal-tonoise ratios $(\mathrm{S} / \mathrm{N})$ of the respective resultant spectra.

The spectra were analyzed using XSPEC version 12.8 .2 (Arnaud 1996). Fits were made across the full NuSTAR band (3-79 keV). Spectra from the FPMA and FPMB were fit jointly, allowing a multiplicative constant to act as a flux normalization factor. The spectra from both observations were grouped to require a minimum of 30 counts per bin to ensure the validity of the $\chi^{2}$ fit statistic (Cash 1979; Gehrels 1986). All errors reported in this work reflect $1 \sigma$ confidence limits.

The $3 \mathrm{keV}$ lower energy limit of NuSTAR is not suited to constraining the column density of the ISM along the line of sight to a source, when its value is low or moderate. A value of
$N_{\mathrm{H}}=7 \times 10^{21} \mathrm{~cm}^{-2}$ is suggested by the HEASARC column density tool, based on Dickey \& Lockman (1990). This value was fixed in all fits to the spectra of GS 1354-645, using the "tbabs" model (Wilms et al. 2000).

We initially considered a canonical spectral model consisting of separate additive components, diskbb (Mitsuda et al. 1984) and a power-law. Neither observation requires the disk blackbody component. It is possible that the disk temperature is simply too low to be detected in the NuSTAR band (see, e.g., Reis et al. 2010; Reynolds \& Miller 2013).

However, the simple power-law component does not achieve a formally acceptable fit to either spectrum. The fit to the lowersensitivity spectrum from Obs. 1 measured a power-law index of $\Gamma=1.369$ (4), but only achieved $\chi^{2} / \nu=2006.50 / 1747=$ 1.149 (where $\nu$ is the number of degrees of freedom in the fit). The spectrum of Obs. 2 is steeper, with $\Gamma=1.528(1)$ in this simple fit, and a very fit statistic result owing to the improved $\mathrm{S} / \mathrm{N}: \chi^{2} / \nu=9904.9 / 2740=3.615$.

These simple fits are shown in Figure 2. The data/model ratio from each fit shows residuals that are consistent with disk reflection, including $\mathrm{Fe} \mathrm{K}$ emission and a Compton backscattering excess peaking in the $20-30 \mathrm{keV}$ range. The ratio from Obs. 2 is consistent with blurred reflection from an inner disk that extends close to the black hole, similar to the features observed in the low/hard state of GRS 1915+105 (see Miller et al. 2013a).

We next considered fits with relxill (version 0.4c; Garcia et al. 2013; Dauser et al. 2014). This model includes the power law that illuminates the disk and also incorporates a Kerr blurring function to translate from the frame of the accretion disk to the frame of the observer. It is this function that measures the strong Doppler shifts and gravitational redshifts imprinted on the reflection spectrum.

Spin is measured directly using relxill, within the bounds $-1 \leqslant a \leqslant 1$. The inclination of the inner disk-not necessarily the same as the binary system-is also measured directly. For these parameters, values obtained in fits to Obs. 2 were assumed in fits to Obs. 1, owing to its lower sensitivity. Relxill also allows the inner disk to deviate from the ISCO for a given spin parameter, so we also allowed the parameter 

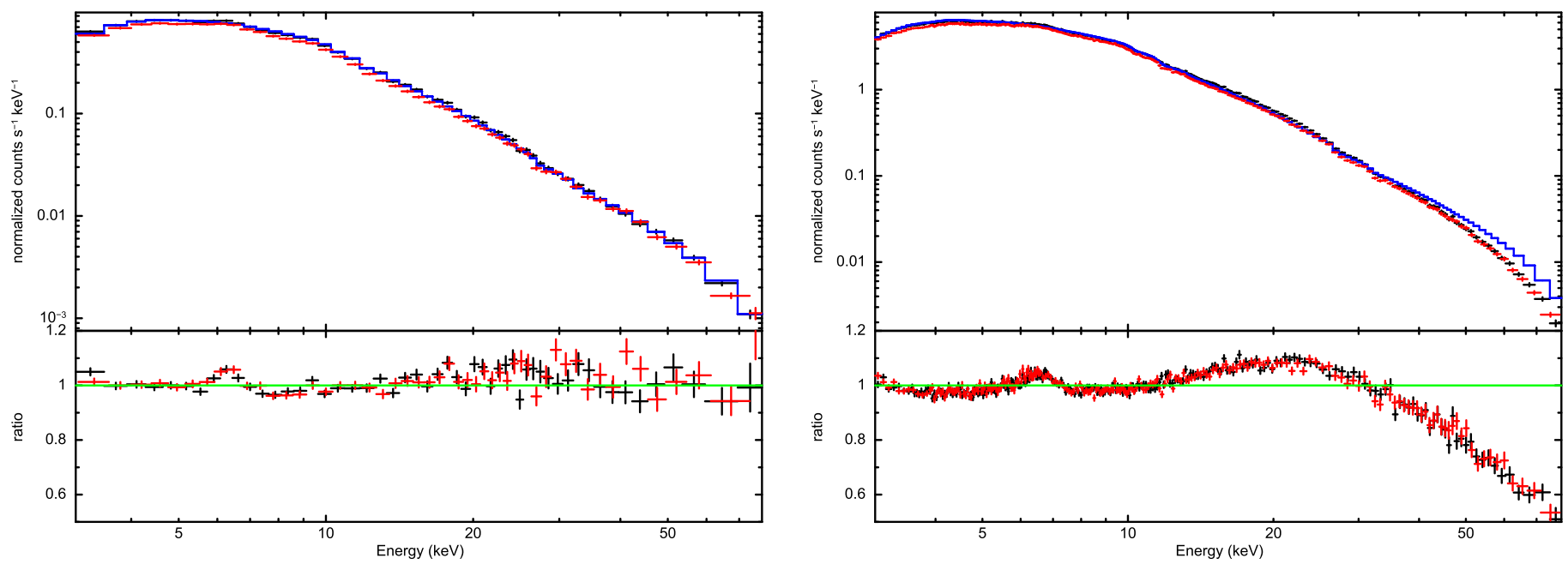

Figure 2. NUSTAR spectra of GS 1354-645, fit with a fiducial power-law continuum. The FPMA is shown in black, the FPMB is shown in red, and the model for the FPMA is shown in blue. The data in each panel have been rebinned for visual clarity. Left: the spectrum of Obs. 1 is shown. Right: the spectrum of Obs. 2 is shown. This ratio reveals evidence of strong, broadened reflection consistent with illumination of the inner accretion disk.

$R_{\text {in }}$ to vary (the model has a hard upper limit of $1000 R_{\mathrm{ISCO}}$ ) in fits to both observations.

The emissivity of the disk is described as a broken power law in radius (e.g., $J \propto r^{-q}$ ), giving three parameters: $q_{\text {in }}, q_{\text {out }}$, and $R_{\text {break. }}$. Any corona with an energetic profile defined by the underlying potential and linked to the disk is likely to have a time-averaged emissivity that falls with radius, so we require $q \geqslant 0$ in fits to both observations. The limited sensitivity of Obs. 1 does not permit more detail to be discerned, so we simply fixed $q_{\text {out }}=q_{\text {in }}$, obviating the meaning of $R_{\text {break }}$. For Obs. 2, however, additional constraints can be imposed based on theoretical and observational expectations. Wilkins \& Fabian (2012) have calculated the emissivity profiles expected for idealized point source scenarios, including a "lamp-post" geometry. All calculations assume a disk that extends to the ISCO. The predicted profiles are complex and a power law with a single break radius is a relatively crude approximation. Inner emissivity profiles steeper than $q=3$ (Euclidean) are only expected in a Kerr geometry, so we have enforced a $R_{\text {break }}<6 G M / c^{2}$ in all fits. The sensitivity afforded by NuSTAR observations of stellar-mass black holes also suggests very small inner disk radii and steep emissivity indices (e.g., Miller et al. 2013a; 2015; Parker et al. 2014; Tomsick et al. 2014).

In addition, we measured the following continuum and reflection parameters using relxill: the power-law index $\Gamma$, the flux normalization of the model, the cutoff energy of the power law $\left(E_{\text {cut }}\right.$; a hard upper limit of $1000 \mathrm{keV}$ is fixed within the model), the reflection fraction ( $f_{\text {reff }}$, the ratio of reflected to incident flux $)$, the iron abundance $\left(A_{\mathrm{Fe}}\right.$; a hard lower limit of $0.5 \leqslant A_{\mathrm{Fe}}$ is fixed within the model), and the ionization of the accretion disk $\left(\xi=L / n r^{2}\right.$, where $L$ is the ionizing luminosity, $r$ is the distance between the source and reflector, and $n$ is the density of the reflecting medium).

The results of fits to the spectra obtained in Obs. 1 and Obs. 2 with relxill are detailed in Table 1 and shown in Figure 3. Owing to the much higher $\mathrm{S} / \mathrm{N}$ of Obs. 2, we fit this spectrum first. The black hole spin, inner disk inclination, and iron abundance measured in Obs. 2 were then frozen in fits to Obs. 1.
Table 1

Spectral Fitting Results

\begin{tabular}{lll}
\hline \hline Parameter & Obs. 1 & Obs. 2 \\
\hline$q_{\text {in }}$ & $1(1)$ & $9(1)$ \\
$q_{\text {out }}$ & $=q_{\text {in }}$ & $0.0^{+0.4}$ \\
$R_{\text {break }}$ & $\cdots$ & $5(1)$ \\
$a\left(\mathrm{cJ} / \mathrm{CM}^{2}\right)$ & $0.998^{*}$ & $0.998_{-0.009}$ \\
$i$ (degrees) & 75 & $75(2)$ \\
$R_{\text {in }}\left(R_{\mathrm{ISCO}}\right)$ & $700_{-500}^{+100}$ & $1.07_{-0.02}^{+0.05}$ \\
$\Gamma$ & $1.46(1)$ & $1.635(7)$ \\
$\log (\xi)$ & $0.3(3)$ & $2.37(3)$ \\
$A_{\mathrm{Fe}}$ & $0.57^{*}$ & $0.57(3)$ \\
$E_{\text {cut }}(\mathrm{keV})$ & $1000_{-200}$ & $150(5)$ \\
$f_{\text {refl }}$ & $0.50(7)$ & $1.5(1)$ \\
Norm. $\left(10^{-3}\right)$ & $3.8(2)$ & $8.4(2)$ \\
$\chi^{2} / \nu$ & $1890.2 / 1742$ & $2753.0 / 2730$ \\
\hline Flux $\left(3-79 \mathrm{keV}, 10^{-9} \mathrm{erg} \mathrm{cm}^{-2} \mathrm{~s}^{-1}\right)$ & $1.01(1)$ & $5.6(1)$ \\
Flux $\left(0.5-100 \mathrm{keV}, 10^{-9} \mathrm{erg} \mathrm{cm}^{-2} \mathrm{~s}^{-1}\right)$ & $1.28(1)$ & $6.9(1)$ \\
\hline
\end{tabular}

Note. The results of spectral fits to NUSTAR observations of the black hole X-ray binary GS 1354-645. The "relxill" model (Garcia et al. 2013; Dauser et al. 2014) was used to describe the combination of the direct and reflected spectra. The fits to Obs. 1 fix the parameters marked with an asterisk at the values measured in Obs. 2. The inner disk inclination is described in terms of $i$. The $R_{\text {in }}$ parameter is measured in units of the ISCO radius for the spin parameter $a$ and allows for the possibility that the disk is not truncated exactly at the ISCO. Please see the text for details concerning the emissivity indices $q$

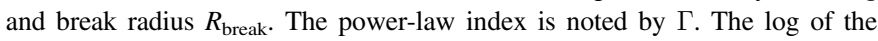
ionization parameter, the abundance of Fe relative to solar, and the power-law cutoff energy are listed as $\log (\xi), A_{\mathrm{Fe}}$, and $E_{\mathrm{cut}}$, respectively. The reflection fraction and flux normalization of "relxill" are listed as $f_{\text {refl }}$ and Norm., respectively. Last, the unabsorbed flux in the spectral fitting band, as well as a broader band, are listed based on these fits.

Far better fits are achieved with relxill $\left(\chi^{2} / \nu=2753.0 /\right.$ $2730=1.008$ for Obs. 2 , and $\chi^{2} / \nu=1890.2 / 1742=1.085$ for Obs. 1). Most importantly, a very high black hole spin parameter is measured in Obs. 2: $a=0.998_{-0.009}$. With the sensitivity of NUSTAR, statistical errors on spin can be small, but systematic errors are likely much larger (see below). The inclination of the inner disk is also very tightly constrained: 

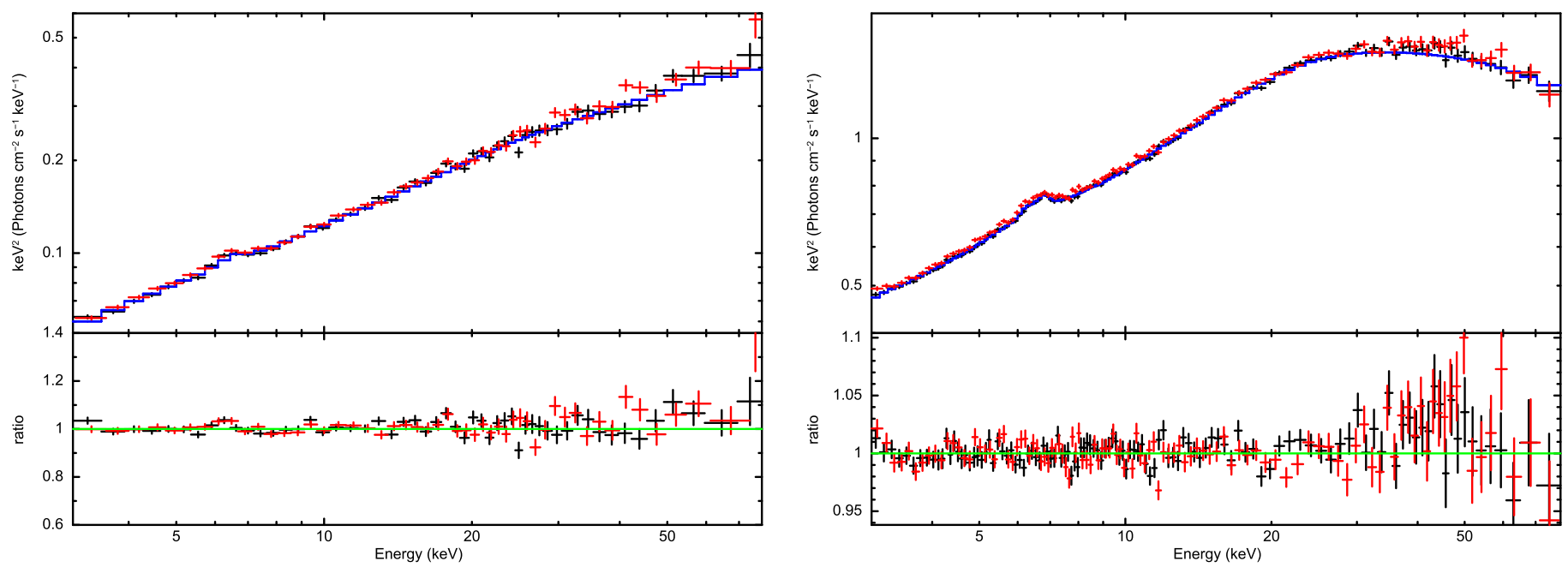

Figure 3. NuSTAR spectra of GS 1354-645, fit with the "relxill" relativistically blurred disk reflection model. The FPMA is shown in black, the FPMB is shown in red, and the model for the FPMA is shown in blue. The data in each panel have been rebinned for visual clarity. Left: the spectrum of Obs. 1 is shown. The data are consistent with reflection from a radially truncated accretion disk. Right: the spectrum of the brighter Obs. 2 is shown. The best fit requires a very high black hole spin parameter.

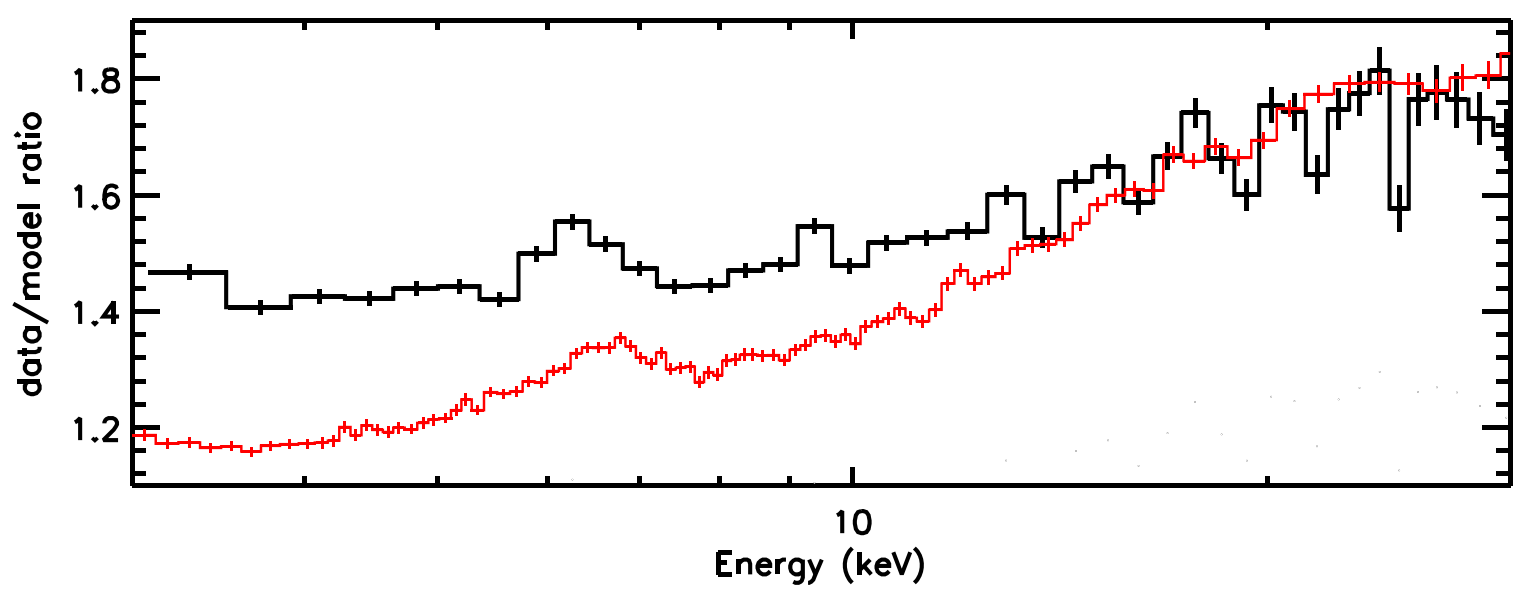

Figure 4. NUSTAR spectra from Obs. 1 (black) and Obs. 2 (red), after removing blurred disk reflection from the models detailed in Table 1. The unmodeled Fe K line in Obs. 2 extends down to $4-5 \mathrm{keV}$, consistent with a disk close to the ISCO around a spinning black hole. In contrast, the unmodeled Fe $\mathrm{K}$ line in Obs. 1 is relatively narrow. Only the FPMA spectra are shown, the ratio from Obs. 1 is multiplied by a factor of 1.4 , and the data were binned for visual clarity.

$i=75(2)$ degrees. Since spin and inclination both work to blur the reflection spectrum, it is important to ensure that the values are not degenerate. Both parameters are tightly constrained, and there appears to be no degeneracy. The inner disk radius is found to be consistent with the ISCO and tightly constrained: $R_{\text {in }}=1.07_{-0.02}^{+0.05} R_{\mathrm{ISCO}}$. This again indicates a disk that extends very close to the black hole.

In contrast to Obs. 2, fits with relxill indicate that the accretion disk did not extend close to the black hole in Obs. 1. The inner disk radius hits the limit of the model, $R_{\mathrm{in}}=700_{-500}^{+200} R_{\mathrm{ISCO}}$. This is indicated by the much narrower line in Obs. 1 (see Figures 2 and 4). The statistical certainty of this result is low; an inner radius of $80 R_{\mathrm{ISCO}}$ is within the $90 \%$ confidence range. We note that the $\mathrm{Fe} \mathrm{K}$ line is not ideally fit, based on the data/model ratio in Figure 3; this may imply that the disk is truncated at a larger radius than allowed within the relxill framework. The addition of a simple Gaussian with an energy fixed at $E=6.40 \mathrm{keV}$ improves the fit at the $3 \sigma$ level of confidence.

Although the reflection fraction in Obs. 1 is lower than in Obs. $2\left(f_{\text {refl }}=0.50(7)\right.$ versus $\left.f_{\text {refl }}=1.5(1)\right)$, it is still fairly high for a compact corona and a much larger inner disk radius. A high reflection fraction and a relatively flat emissivity index in Obs. 1 may be nominally consistent with a large corona that partially blankets the disk, but a standard emissivity profile $(q=3)$ is allowed by the data at the $90 \%$ confidence level.

\section{DISCUSSION AND CONCLUSIONS}

We have analyzed two NUSTAR spectra of the dynamically confirmed black hole GS 1354-645. Both observations were obtained in the "low/hard" state. When fit with a relativistic reflection model, the spectrum obtained close to the outburst peak suggests a high spin parameter and also implies that the inner disk may be viewed at a high inclination. The spectrum obtained at a lower flux level suggests that the inner disk may have been truncated, potentially consistent with radiatively inefficient accretion flow models. In this section, we discuss the strengths and weaknesses of our results, potential sources of systematic errors, and impacts on our understanding of GS 1354-645.

Fits to Obs. 2 with relxill indicate that the accretion disk likely extends very close to the black hole. This is now 
common in the most luminous phases of the "low/hard" state, especially at the sensitivity afforded by $N u S T A R$ (see, e.g., Miller et al. 2015). The data strongly require a very high spin parameter, $a=0.998_{-0.009}$, consistent with the extreme upper bound of the model. The error is merely the statistical error, and systematic errors are likely to be much larger.

All measures of black hole spin obtained through the accretion disk rely on the optically thick reflecting gas obeying the test particle ISCO. Simulations suggest that the disk is likely to be thin and to obey the ISCO at Eddington fractions below 0.3 (Reynolds \& Fabian 2008; Shafee et al. 2008); for plausible combinations of black hole mass and distance, this condition was met in our observations. It is quite possible, however, that no astrophysical disk respects the ISCO at the percent level.

The best-fit model for Obs. 2 yields parameters similar to those expected in a "lamp-post" geometry (a compact, central source of hard X-rays located on the spin axis above the black hole). The relxilllp model (Garcia et al. 2013; Dauser et al. 2014) explicitly assumes this geometry and calculates the reflection fraction self-consistently; it also gives a high spin parameter $(a>0.85)$. However, our best-fit model (see Table 1) is superior at the $6.6 \sigma$ level of confidence, as determined by an F-test (for relxilllp, $\chi^{2} / \nu=2806$ / 2733 , even if the reflection fraction is not linked to the lamppost value). This might imply that the corona is indeed compact but not quite an idealized lamp-post. Recent work has noted some physical difficulties with idealized lamp-post models (e.g., Niedzwiecki et al. 2016; Vincent et al. 2016).

Stiele \& Kong (2016) have reported a nearly maximal retrograde spin based on a short XMM-Newton observation of GS 1354-645. A combination of factors including calibration uncertainties in the EPIC-pn camera and photon pile-up may have acted to falsely narrow the reflection features in the XMMNewton spectrum (Miller et al. 2010). It is also possible that the disk was mildly truncated during the XMM-Newton observation. Fits to NuSTAR Obs. 2 with $a \leqslant 0.93$ are rejected at the $5 \sigma$ level of confidence, via an F-test.

Our reflection modeling also indicates that the inner disk is viewed at a relatively high inclination, $i=75(2)$ degrees. This is within the eclipse limit derived by Casares et al. (2009). It is possible that the inner disk is not aligned with the inclination of the binary system itself (Maccarone 2002; also see Tomsick et al. 2014).

Systems that narrowly avoid eclipses are often observed to exhibit "dips" in their X-ray light curve. These events may be due to vertical structures in the outer accretion disk that block some of the light from the central engine (see, e.g., Diaz-Trigo et al. 2006). We did not detect any dips in the light curve of GS 1354-645, possibly indicating that the inclination of the outer disk must really be lower than the value derived for the inner disk via reflection. However, dips may only be manifested at higher Eddington fractions (see, e.g., Kuulkers et al. 2000) than the luminosities inferred in our NUSTAR exposures.

Esin et al. (1997) predict that the inner accretion flow will become advection-dominated and radiatively inefficient at Eddington fractions below 0.01 . In the $0.01-0.08 L_{\text {Edd }}$ range, the inner disk may still be truncated but the inner flow can be more luminous. For GS 1354-645, assuming a distance at the lower limit of $d=25 \mathrm{kpc}$ and mass at the lower limit of $M=7.6 M_{\odot}$, the luminosities based on the flux values in Table 1 would be $0.1 L_{\text {Edd }}$ and $0.53 L_{\text {Edd }}$ for Obs. 1 and Obs. 2, respectively. Smaller distances $(d<10 \mathrm{kpc})$ would more easily accommodate the lower end of the luminosity range at which thin disks may truncate. Alternatively, some combinations including very high black hole masses $\left(M>90 M_{\odot}\right)$ can also meet the prediction, but these prescriptions greatly exceed the range of black hole masses inferred in X-ray binaries (e.g., Remillard \& McClintock 2006).

We thank the anonymous referee for comments that improved this manuscript. This work was supported under NASA contract No. NNG08FD60C, and made use of data from the NuSTAR mission, a project led by the California Institute of Technology, managed by the Jet Propulsion Laboratory, and funded by NASA.

\section{REFERENCES}

Arnaud, K. 1996, in ASP Conf. Ser. 101, Astronomical Data Analysis Software and Systems V, ed. G. H. Jacoby \& J. Barnes (San Francisco, CA: ASP), 17 Bardeen, J., Press, W., \& Teukolsky, S. 1972, ApJ, 178, 347

Brocksopp, C., Jonker, P. G., Fender, R. P., et al. 2001, MNRAS, 323, 517

Casares, J., Orosz, J. A., Zurita, C., et al. 2009, ApJ, 181, 238

Cash, W. 1979, ApJ, 228, 939

Dauser, T., Garcia, J., Parker, M., Fabian, A., \& Wilms, J. 2014, MNRAS, 444, L100

Diaz-Trigo, M., Parmar, A., Boirin, L., Mendez, M., \& Kaastra, J. 2006, A\&A, 445,179

Dickey, J. M., \& Lockman, F. J. 1990, ARA\&A, 28, 215

Esin, A. A., McClintock, J. E., \& Narayan, R. 1997, ApJ, 489, 865

Furst, F., Nowak, M. A., Tomsick, J. A., et al. 2015, ApJ, 808, 122

Garcia, J., Dauser, T., Reynolds, C., et al. 2013, ApJ, 768, 146

Gehrels, N. 1986, ApJ, 303, 336

Harrison, F. A., Craig, W. W., Chrstensen, F. E., et al. 2013, ApJ, 770, 103

King, A. L., Walton, D. J., Miller, J. M., et al. 2014, ApJL, 784, L2

Kitamoto, S., Tsunemi, H., Pederson, H., Ilovaisky, S., \& van der Klis, M. 1990, ApJ, 361, 590

Kuulkers, E., in't Zand, J. J. M., Conelisse, R., et al. 2000, A\&A, 358, 993

Maccarone, T. 2002, MNRAS, 336, 1371

Miller, J. M. 2007, ARA\&A, 45, 441

Miller, J. M., D’Ai, A., Bautz, M., et al. 2010, ApJ, 724, 1441

Miller, J. M., Parker, M. L., Fuerst, F., et al. 2013a, ApJL, 775, L45

Miller, J. M., Parker, M. L., Fuerst, F., et al. 2013b, ApJL, 779, L2

Miller, J. M., Reynolds, M. T., \& Kennea, J. 2015, ATel, 7612

Miller, J. M., Tomsick, J. A., Bachetti, M., et al. 2015, ApJL, 799, L6

Miller, M. C., \& Miller, J. M. 2015, PhR, 548, 1

Mitsuda, K., Inoue, H., Koyama, K., et al. 1984, PASJ, 37, 741

Niedzwiecki, A., Zdziarski, A., \& Szanecki, M. 2016, ApJL, 821, L1

Parker, M. L., Wilkins, D. R., Fabian, A. C., et al. 2014, MNRAS, 443, 1723

Reis, R., Fabian, A., \& Miller, J. M. 2010, MNRAS, 402, 836

Remillard, R. A., \& McClintock, J. E. 2006, ARA\&A, 44, 49

Revnivtsev, M., Borozdin, K., Priedhorsky, W., \& Vikhlinin, A. 2000, ApJ, 530, 955

Reynolds, C. 2014, SSRv, 183, 277

Reynolds, C., \& Fabian, A. C. 2008, ApJ, 675, 1048

Reynolds, M., \& Miller, J. M. 2013, ApJ, 769, 16

Risaliti, G., Harrison, F. A., Madsen, K. K., et al. 2013, Natur, 494, 449

Shafee, R., Narayan, R., \& McClintock, J. 2008, ApJ, 676, 549

Stiele, H., \& Kong, A. 2016, MNRAS, 459, 4038

Tomsick, J. A., Nowak, M. A., Parker, M., et al. 2014, ApJ, 780, 78

Vincent, F. H., Rozanska, A., Zdziarski, A. A., \& Madej, J. 2016, A\&A, 590, 132

Wilkins, D., \& Fabian, A. 2012, MNRAS, 424, 1284

Wilms, J., Allen, A., \& McCray, R. 2000, ApJ, 542, 914 